\title{
“Maestra Delivery" y el desarrollo de la grafomotricidad en niños, Ilave-(Perú) 2021
}

\author{
"Maestra Delivery" and the development of graphomotricity in children, Ilave- (Peru) 2021
}

"Maestra Delivery" e o desenvolvimento de habilidades grafomotoras em crianças, Ilave- (Peru) 2021

\section{Yolynda Maquera Maquera \\ yolynda34@gmail.com \\ https://orcid.org/0000-0002-9018-846X}

Universidad Nacional del Altiplano, Puno, Perú

\section{Yuselino Maquera Maquera}

ymmaquera@unap.edu.pe

https://orcid.org/0000-0002-7476-9205

Universidad Nacional del Altiplano, Puno, Perú

\section{RESUMEN}

El objetivo de la investigación fue determinar en qué medida la estrategia aprendizaje - servicio "maestra delivery" mejora el desarrollo de la grafomotricidad en niños en edad preescolar. La metodología fue cuantitativa, de tipo experimental, diseño cuasi experimental, con una población de 53 niños. Se aplicó una pre y post prueba. Los resultados se procesaron en el programa STATA16, la muestra fue una proporción considerable de $74 \%$; con la prueba Dunnet, media aritmética _n la prueba de entrada es de $\bar{x}=10.3$ y en la Prueba de Salida $\bar{x}_{\mathrm{s}}^{\mathrm{e}}=16.4$ de competencias desarrolladas en actividades de grafomotricidad con el test de comparación de medias a través de las escalas AD Logro destacado (2018), A Logro (17 -14), B Proceso (13 -11) y C Inicio (10-0). Se concluyó que la estrategia delivery en tiempos de pandemia es eficiente, pues desarrolla habilidades de grafomotricidad en niños en edad preescolar, con talleres de aprendizaje.

Palabras clave: Maestra delivery; Estrategia; Grafomotricidad; pandemia

\section{ABSTRACT}

The objective of the research was to determine to what extent the "teacher delivery" service-learning strategy improves the development of graphomotor skills in preschool children. The methodology was quantitative, experimental, quasi-experimental design, with a population of 53 children. A pre and post test was applied. The results were processed in the STATA16 program, the sample was a considerable proportion of $74 \%$; With the Dunnet test, the arithmetic mean in the entrance test is $\mathrm{x} \mathrm{e}=10.3$ and in the Exit Test $\mathrm{x}$ $s=16.4$ of competencies developed in graphomotor activities with the test of comparison of means through the $\mathrm{AD}$ scales. Outstanding achievement (2018), A Achievement (17 -14), B Process (13 -11) and C Start (10-0). It was concluded that the delivery strategy in times of pandemic is efficient, since it develops graphomotor skills in preschool children, with learning workshops.

Key words: Delivery teacher; Strategy; Graphomotor Skills; pandemic

\section{RESUMO}

O objetivo da pesquisa foi determinar em que medida a estratégia de aprendizagem de serviço "entrega do professor" melhora o desenvolvimento das habilidades grafomotoras em crianças pré-escolares. A metodologia foi quantitativa, experimental, com delineamento quase experimental, com uma população de 53 crianças. Um pré e pós-teste foi aplicado. Os resultados foram processados no programa STATA16, a amostra foi uma proporção considerável de 74\%; Com o teste de Dunnet, a média aritmética no teste de entrada é x e = 10,3 e no Teste de Saída x $s=16,4$ das competências desenvolvidas nas atividades grafomotoras com o teste de comparação de médias por meio das escalas AD.), A Realização (17-14), Processo B (13 -11) e C Iniciar (10-0). Concluiu-se que a estratégia de entrega em tempos de pandemia é eficiente, uma vez que desenvolve habilidades grafomotoras em pré-escolares, com oficinas de aprendizagem.

Palavras-chave: Professor de deliberação; estratégia; habilidades grafomotoras; pandemia 


\section{INTRODUCCIÓN}

El aprendizaje en servicio (A-S) es considerado como una propuesta pedagógica, programa, estrategia (Mendía, 2012; Puig et al. 2007), que combina de manera activa y participativa procesos de aprendizaje y servicio a la comunidad, aportando un escenario formativo que potencia las competencias de comunicación, actitudes sociales y la motivación re- querida para la concreción de las tareas y competencias pre- vistas (Chiva-Bartoll et al. 2018; Gil et al. 2016; Huda et al., 2018). Entre los rasgos a destacar del A-S, según Mayor y Rodríguez (2015), están el servicio a la comunidad con la intención de mejorarla, la participación activa de las personas implicadas y la planificación intencional de los objetivos curriculares y las acciones que conforman el servicio.

La educación inicial, a diferencia de otros niveles de educación básica, no enseña contenidos temáticos, ni trata a los niños como estudiantes en situación escolar.Sin embargo, las posibilidades de un aprendizaje eficiente de la escritura, frecuentemente, son limitadas en los entornos preescolares (Gerde et al. 2019); persisten las prácticas pedagógicas rutinarias que siguen un itinerario mecánico, preestablecido, poco propicio para enriquecer la actuación y configuración del andamiaje de la expresión y comunicación lingüística, que debe fundamentarse en el conocimiento metalingüístico (Arnaíz y Bolarín, 2016), como capacidad para reconocer la naturaleza, formas y funciones del lenguaje escrito.

En tal sentido, el objetivo de la investigación fue demostrar la eficiencia de la "maestra delivery", a través de talleres para desarrollar grafomotricidad en niños por constituir el primer nivel de la escritura. Se justifica el presente estudio, pues crear alternativas de respuesta a necesidades y problemas complejos de los niños preescolares, exige a las maestras, cada vez, mayor adaptabilidad e innovación en el empleo de diversas estrategias comunicativas en todo episodio interactivo, variando de forma cualitativa y cuantitativa (Gonzáles, 2015). De lo contrario, el fracaso en la alfabetización temprana de los niños preescolares, se mantiene invariablemente, $y$ cuando esto ocurre, se pierde el objetivo del aprendizaje de la escritura como una "herramienta de vida de cada ser humano y pasa a convertirse en un problema de aprendizaje" (Suárez, 2004, p. 6), que obstruye y retarda el proceso de aprendizaje.

\section{Características del aprendizaje-servicio}

A partir de Puig y Palos (2006) se establecen seis características del aprendizaje de servicio:

1. Es un método apropiado para la educación formal y no formal, válido para todas las edades $y$ aplicable en distintos espacios temporales

Se aplican en ámbitos educativos formales y no formales, a cualquier edad y en todos los niveles educativos. El proyecto o experiencia de aprendizaje debe ser de acuerdo a las características, necesidades y contexto del estudiante.

2. Se propone llevar a cabo un servicio auténtico a la comunidad que permita aprender $y$ colaborar en un marco de reciprocidad

El aprendizaje- servicio encierra, como la misma palabra lo indica, el servicio como una respuesta a necesidades reales de los estudiantes, familia y comunidad. El servicio permite lograr competencias en los estudiantes, a través de esfuerzo, organización y cooperación de la familia, una oportunidad para el ejercicio de la responsabilidad y, sobre todo, un espacio de 
colaboración recíproca donde todos los agentes educativos participan.

3. Desencadena procesos sistemáticos y ocasionales de adquisición de conocimientos y competencias para la vida

Las propuestas de aprendizaje - servicio persiguen desarrollar un trabajo explícito para el logro de los aprendizajes, a través de los procesos conscientes, planificados y sistemáticos de enseñanza - aprendizaje, que relacionan las tareas de servicio con contenidos y el desarrollo de competencias relevantes para la vida.

4. Supone una pedagogía de la experiencia y la reflexión

El aprendizaje-servicio está en contra de una pedagogía tradicional y memorística. Por el contrario, se inspira en una pedagogía que parte por los principios de experiencia o las necesidades, intereses y contexto del estudiante. En las diferentes fases del proyecto o vivencias de aprendizaje se requiere la participación activa del estudiante, la reflexión, cooperación o trabajo conjunto y colaboración de los padres de familia, la evaluación es permanente permite la reflexión de los participantes y el proyecto tiene como fin resolver problemas. Una pedagogía de estas características requiere que el educador sea mucho más que un enseñante.

5. Requiere una red de alianzas entre las instituciones educativas $y$ las entidades sociales que facilitan servicios a la comunidad El aprendizaje - servicio depende de una imagen de la escuela que rompa con la tendencia a encerrarse en sí misma. El aprendizaje servicio requiere por naturaleza un trabajo en red que coordine las instituciones educativas y las entidades sociales que facilitan la intervención en la realidad. Esta alianza permitirá a las instituciones educativas abrirse a su entorno, y a las entidades sociales ejercer, además de las tareas que les son propias, una influencia formativa que complete la acción de las instituciones educativas y contribuya así al ideal de la ciudad educadora.

6. Provoca efectos en el desarrollo personal, cambios en las instituciones educativas y sociales que lo impulsan, y mejoras en el entorno comunitario que recibe el servicio

El aprendizaje-servicio es una práctica educativa que ayuda a los estudiantes a adquirir conocimientos y procedimientos curriculares relacionados con el servicio, desarrolla capacidades, competencias que inducen a la resolución de problemas. Pero, además, suele incidir en la motivación, aumenta la autoestima y las expectativas personales, y hace más realistas y esperanzados a los participantes.

El aprendizaje-servicio es, adicionalmente, una actividad fundamental para la educación en valores, forma la responsabilidad, desarrolla la identidad y autonomía en los estudiantes.

\section{MÉTODO}

El estudio se correspondió con el método deductivo-inductivo, paradigma cuantitativo, de corte longitudinal, su diseño fue cuasiexperimental con un solo grupo, con pre y post prueba; se consideró como variable independiente la estrategia de aprendizaje-servicio que utiliza la “maestra delivery". Se ejecutó en el primer semestre del año escolar 2021, en las Instituciones Educativas Iniciales (IEIs) de Checca, Laccaya, Yaurima y Simillaca, jurisdicción del distrito de Ilave, provincia El Collao-Ilave, departamento de Puno (Perú). Cuya población es predominantemente aimara hablante. 
Participaron el total de niños y padres de familia, sección única de 05 años: a) 53 niños (30 varones y 23 mujeres) yb) 53 padres de familia (40 mujeres y 13 varones). Para la selección de la población primaron 2 criterios: la predisposición y cooperación de la maestra de aula y el consentimiento informado e involucramiento real de los padres de familia en la propuesta pedagógica, en una coyuntura muy especial, educación remota en tiempos de pandemia Covid-19. (Tabla 1).

Tabla 1. Población de estudio según Instituciones Educativas Iniciales en provincia el Collao y Departamento de Puno (Perú).

\begin{tabular}{ccccc}
\hline Nro. & INSTITUCIONES EDUCATIVAS INICIALES & NIÑOS & TOTAL \\
\hline 1 & IEI 319 Checca & 11 & 11 \\
2 & IEI 280 Laccaya & 18 & 18 \\
3 & IEI 268 Yaurima & 13 & 13 \\
4 & IEI 225 Simillaca & & 11 & 11 \\
& & TOTAL & $\mathbf{5 3}$ & $\mathbf{5 3}$ \\
\hline
\end{tabular}

En contextos rurales de población aimara, se espera de la escuela una apertura permanente para articular expectativas y formas de vida de la población usuaria con las actividades escolares. No obstante, en la institución educativa seleccionada para este trabajo, se advirtió la existencia de diferentes problemas y necesidades que ponen en evidencia la ausencia y precariedad de la organización escolar para ofrecer respuestas a las demandas sociales y educativas particulares. Los padres se mostraron cada vez más insatisfechos y exigentes, respecto al desempeño de las maestras y el aprendizaje alcanzado por los niños. Ante estas circunstancias evidentes, se ideó la estrategia de aprendizaje-servicio, denominado "maestra delivery", que involucra tanto a padres como a la maestra de aula, para conjuntamente colaborar en el aprendizaje de las habilidades grafomotoras, en niños en edad preescolares.

La metodología empleada corresponde a la investigación cuantitativa, diseño cuasiexperimental con un solo grupo, con pre y post prueba; se consideró como variable independiente la estrategia de aprendizaje-servicio que utiliza la "maestra delivery", y como dependiente el desarrollo de la talleres de aprendizaje de grafomotricidad, que tuvo por dimensiones de estudio a los niveles: manipulativo-vivencial (trazos sincréticos tensos y distendidos); interiorización simbólica (trazos lineales tensos y distendidos); y representación perceptiva (trazos iconográficos y realización de opacidades), que fueron medidos por la escala preestablecidas por el Ministerio de Educación del Perú: Inicio (00-10), Proceso (11-13) y Logro (16 -17) Logro destacado (18-20) y los datos fueron analizados mediante la prueba " $\mathrm{t}$ " de diferencias de medias con un grupo en dos tiempos al 95\% de confiabilidad. Aplicados y procesados con STATA 16.

El programa Talleres de Aprendizaje o experiencia de aprendizaje se ejecutó durante el primer semestre del año escolar 2021, en los horarios habituales de clase entre lunes a viernes en aula y 02 horas por semana en cada casa u hogar, 
en horarios variables y concertados con los padres de familia. Durante los procesos de planificación, ejecución y evaluación del aprendizaje de las competencias de grafomotricidad por los niños en edad pre escolar, participaron colaborativamente maestras y padres de familia, cuidando que las actividades estén contempladas en el marco curricular correspondiente. La ejecución de las actividades de aprendizaje se establece en tiempos de Covid19, donde existe problemas de conectividad por el programa remoto "Aprendo en Casa" y donde la "maestra delivery" tiene caminar de casa para lograr los objetivos educaciones en tiempos de confinamiento, tanto en aula como en el hogar, fueron definidas de manera dialógica y participativa entre maestras y padres de familia; especificando qué acciones deben desarrollarse en aula y cuáles a cargo de los padres de familia con apoyo y asistencia de la "maestra delivery".

Los roles de la "maestra delivery" consistieron básicamente en: a) establecer horarios de trabajo concertados, preveer recursos de aprendizaje (sogas, aros, balones, lápices de color, papel, tijera, cintas y otros materiales educativos contemplados en los talleres de aprendizaje); guiar, brindar pautas específicas a los padres o familiares del niño, para llevar adelante las actividades programadas en casa (casa andina), cuidando que estén en el sentido de los propósitos de aprendizaje establecidos y b) constituirse en el hogar de los niños para brindar asistencia-asesoramiento oportunos, según circunstancias o necesidades de intervención, además de reemplazar a los padres en casos requeridos. Finalmente, el logro de los aprendizajes en grafomotricidad fueron determinados a través de los resultados obtenidos mediante la post prueba y de más pruebas estadísticas aplicadas en el programa estadístico STATA16, a fin de determinar prueba de t de diferencia de medias, la prueba de hipótesis y la prueba Dunnet de comparación proporcional.

\section{RESULTADOS Y DISCUSIÓN}

\section{Desarrollo de competencias grafomotoras mediante aprendizajes-servicio: "maestra delivery"}

Los hallazgos en el estudio evidencian cambios sustanciales respecto a los efectos que generan las actividades de aprendizaje en el desarrollo de competencias grafomotoras. Se consolida la estrategia "maestra delivery", la eficiencia de la estrategia "maestra delivery" en tiempos de pandemia ha jugado un papel importante de visitar de casa en casa, ya que existe brechas respecto al acceso a la tecnología como la Plataforma Google Meet, Zoom, Webex y realizar las acciones de réplica de la estrategia "aprendo en casa" emitida por el canal del Estado peruano, no llega eficientemente a la provincia de El Collao, Ilave, por problemas de conectividad y acceso a la tecnología. Esta pandemia del Covid-19, ha puesto en evidencia cómo el sistema educativo, no responde a las grandes exigencias de la modernidad y el uso de la tecnología.

En este contexto, para seguir adelante con los procesos de enseñanza aprendizaje en el nivel inicial, muchas maestras tuvieron que recurrir al ingenio, a la revisión de literatura y experiencias en España, la enseñanza delivery, y a las redes de la UGEL El Collao. Las docentes han optado por diferentes estrategias con el fin de encarar la pandemia, pues la educación no puede parar.

Por tanto, la reflexión cabe ¿es posible innovar estrategias de enseñanza en tiempo de pandemia en el nivel inicial? Contundentemente, a partir de la presente investigación se responde que sí. El argumento se da porque la tarea consiste en trabajar con la familia para mejorar los aprendizajes, en respuesta a esta pandemia. La eficiencia y efectividad de la estrategia "maestra delivery", para el desarrollo de competencias grafomotoras a través de talleres en niños de educación inicial. Es preciso acotar 
que se ejecutó la prueba y post prueba durante el tratamiento experimental con un solo grupo, cuyos resultados se ofrece a continuación.

\section{Evaluación inicial}

Realizando el análisis estadístico, se determinó la sumatoria, la media aritmética de los niveles de grafomotricidad, mediante la prueba de $t$ test de medias comparadas. (Tabla 2) indica que tiene un valor aritmético de ${ }_{\mathrm{e}}^{\bar{x}}=10.3$, y en términos literales se encuentran en el nivel de inicio, con el $43 \% y$ proceso con el $45 \%$ respectivamente.
El resultado obtenido es de 10.3, el mismo indica que los niños de educación inicial antes del tratamiento experimental de los talleres de aprendizaje se encuentran mayoritariamente en la Escala Inicio y Proceso, respecto al desarrollo de competencias grafomotoras. Implicó que los niños objetos de estudio presentaron dificultades al realizar trazos sincréticos, trazos lineales tensos, trazos lineales distendidos, trazos iconográficos y opacidades. Es evidente, que no ha desarrollado competencias motrices y no son las más adecuadas, en la relación a la edad que pertenecen los niños.

Tabla 2. Resultados de las competencias grafomotoras en niños de educación inicial en la pre prueba.

\begin{tabular}{|c|c|c|c|c|c|c|c|c|c|}
\hline \multirow{2}{*}{ Talleres de aprendizaje } & \multirow{2}{*}{$\begin{array}{l}\text { Indicadores de la } \\
\text { grafomotricidad }\end{array}$} & \multicolumn{2}{|c|}{ Inicio } & \multicolumn{2}{|c|}{ Proceso } & \multicolumn{2}{|c|}{ Logro } & \multicolumn{2}{|c|}{$\begin{array}{c}\text { Logro } \\
\text { Destacado }\end{array}$} \\
\hline & & f & $\%$ & f & $\%$ & f & $\%$ & f & $\%$ \\
\hline $\begin{array}{l}\text { Realizamos el recorrido del } \\
\text { carrito }\end{array}$ & Trazos sintéticos & 23 & $43 \%$ & 25 & $47 \%$ & 5 & $9 \%$ & & \\
\hline $\begin{array}{l}\text { Realizamos el recorrido de } \\
\text { las olas }\end{array}$ & Trazos sintéticos & 23 & $43 \%$ & 22 & $42 \%$ & 8 & $15 \%$ & & \\
\hline $\begin{array}{l}\text { Formamos unas figuras } \\
\text { geométricas }\end{array}$ & Trazos Lineales Tensos & 24 & $45 \%$ & 24 & $45 \%$ & 5 & $9 \%$ & & \\
\hline Jugando con mi cuerpo & Trazos Lineales Distendidos & 30 & $57 \%$ & 22 & $42 \%$ & 1 & $2 \%$ & & \\
\hline Realicemos figuras abiertas & Trazos iconográficos & 28 & $53 \%$ & 22 & $42 \%$ & 5 & $9 \%$ & & \\
\hline \multirow{4}{*}{$\begin{array}{l}\text { Realizamos figuras con } \\
\text { nuestro cuerpo }\end{array}$} & Opacidades & 11 & $21 \%$ & 30 & $57 \%$ & 12 & $23 \%$ & & \\
\hline & Sumatoria & 139 & & 145 & & 36 & & & \\
\hline & Promedio & 23.167 & & 24.167 & & 6 & & & \\
\hline & $\%$ & 23 & 43 & 24 & $45 \%$ & 6 & $11 \%$ & & \\
\hline
\end{tabular}

\section{Eficiencia de los talleres de grafomotricidad}

El promedio alcanzado, ${ }_{\mathrm{s}}^{\overline{\mathrm{s}}}=16.4$, señala que los niveles de aprendizaje logrado por los niños, después del proceso de tratamiento, con el desarrollo de competencias en grafomotricidad y talleres de aprendizaje y la estrategia la "maestra delivery" en tiempos de pandemia COVID, la maestra de educación inicial ha tenido que visitar de casa en casa, para cumplir sus objetivos, alcanzando que los niños, producto del tratamiento experimental, 
se encuentren en su mayoría en la Escala Logro y logro destacado, con indicadores establecidos por el Ministerio de Educación (Perú). Con el 64 y 36 $\%$ respectivamente. Los niños superan las barreras iniciales y se encuentran con una actitud proactiva y se encuentran con una actitud consciente y reflexiva de realizar trazos sincréticos, trazos lineales tensos, trazos lineales distendidos, trazos iconográficos y opacidades. Es evidente, que no ha desarrollado competencias motrices y no son las más adecuadas, en la relación a la edad que pertenecen los niños. En efecto, los talleres de aprendizaje individual de casa en casa tuvieron eficiencia en relación a la media aritmética de la pre-prueba, las competencias grafomotrices se desarrollaron también con el apoyo de los padres de familia. (Tabla 3).

Tabla 3. Resultados de las competencias grafomotoras en niños de educación inicial en la post prueba.

\begin{tabular}{|c|c|c|c|c|c|c|c|c|c|}
\hline \multirow[t]{2}{*}{ Talleres de aprendizaje } & \multirow{2}{*}{$\begin{array}{l}\text { Indicadores de la } \\
\text { grafomotricidad }\end{array}$} & \multicolumn{2}{|c|}{ Inicio } & \multicolumn{2}{|c|}{ Proceso } & \multicolumn{2}{|c|}{ Logro } & \multicolumn{2}{|c|}{$\begin{array}{c}\text { Logro } \\
\text { Destacado }\end{array}$} \\
\hline & & f & $\%$ & $\mathbf{f}$ & $\%$ & f & $\%$ & f & $\%$ \\
\hline $\begin{array}{l}\text { Realizamos el recorrido del } \\
\text { carrito }\end{array}$ & Trazos sintéticos & & & & & 33 & $62 \%$ & 20 & $38 \%$ \\
\hline $\begin{array}{l}\text { Realizamos el recorrido de } \\
\text { las olas }\end{array}$ & Trazos sintéticos & & & & & 37 & $70 \%$ & 16 & $30 \%$ \\
\hline $\begin{array}{l}\text { Formamos unas figuras } \\
\text { geométricas }\end{array}$ & Trazos Lineales Tensos & & & & & 29 & $55 \%$ & 24 & $45 \%$ \\
\hline Jugando con mi cuerpo & Trazos Lineales Distendidos & & & & & 35 & $66 \%$ & 18 & $34 \%$ \\
\hline Realicemos figuras abiertas & Trazos iconográficos & & & & & 38 & $72 \%$ & 15 & $28 \%$ \\
\hline \multirow{4}{*}{$\begin{array}{l}\text { Realizamos figuras con } \\
\text { nuestro cuerpo }\end{array}$} & Opacidades & & & & & 34 & $64 \%$ & 19 & $36 \%$ \\
\hline & Sumatoria & & & & & 206 & & 112 & \\
\hline & Promedio & & & & & 34.333 & & 18.667 & \\
\hline & $\%$ & & & & & 34 & $64 \%$ & 19 & $36 \%$ \\
\hline
\end{tabular}

Los padres de familia cumplieron un papel decisivo, constituyeron el nuevo hallazgo no previsto en el estudio, que se enuncia para ampliar esta variable de investigación. En el hogar de las familias de extracción campesina, los padres colaboran con la ejecución de tareas de los talleres, a partir del juego, con secuencias logran las competencias rafomotrices en los niveles de manipulaciónvivencial, el proceso de asimilación simbólica y las representaciones perceptivas, en niños antes del proceso de educación primaria.

En términos generales, el estudio confirmó la eficiencia y eficacia, en tiempos de pandemia, de la intervención de la estrategia de la "maestra delivery", y el cumplimiento de propósitos socioculturales en tiempos de educación remota y en el marco de la formación aprendo en casa de educación inicial. 


\section{La prueba estadística}

Los resultados obtenidos mediante STATA respecto a la media aritmética en la prueba de entrada son de ${ }_{\mathrm{e}}^{\bar{x}}=10.3$ y en la Prueba de Salida $\bar{x}$ $=16.4$, en términos estrictamente aritméticos. Y con datos no agrupados. Asimismo, en el Gráfico
1, se presenta, en comportamiento de los 53 niños, por logros de grafomotricidad en tres tiempos, pre prueba - proceso y post prueba. Y se puede declarar eficiente el programa por la línea verde respecto al punto inicial.

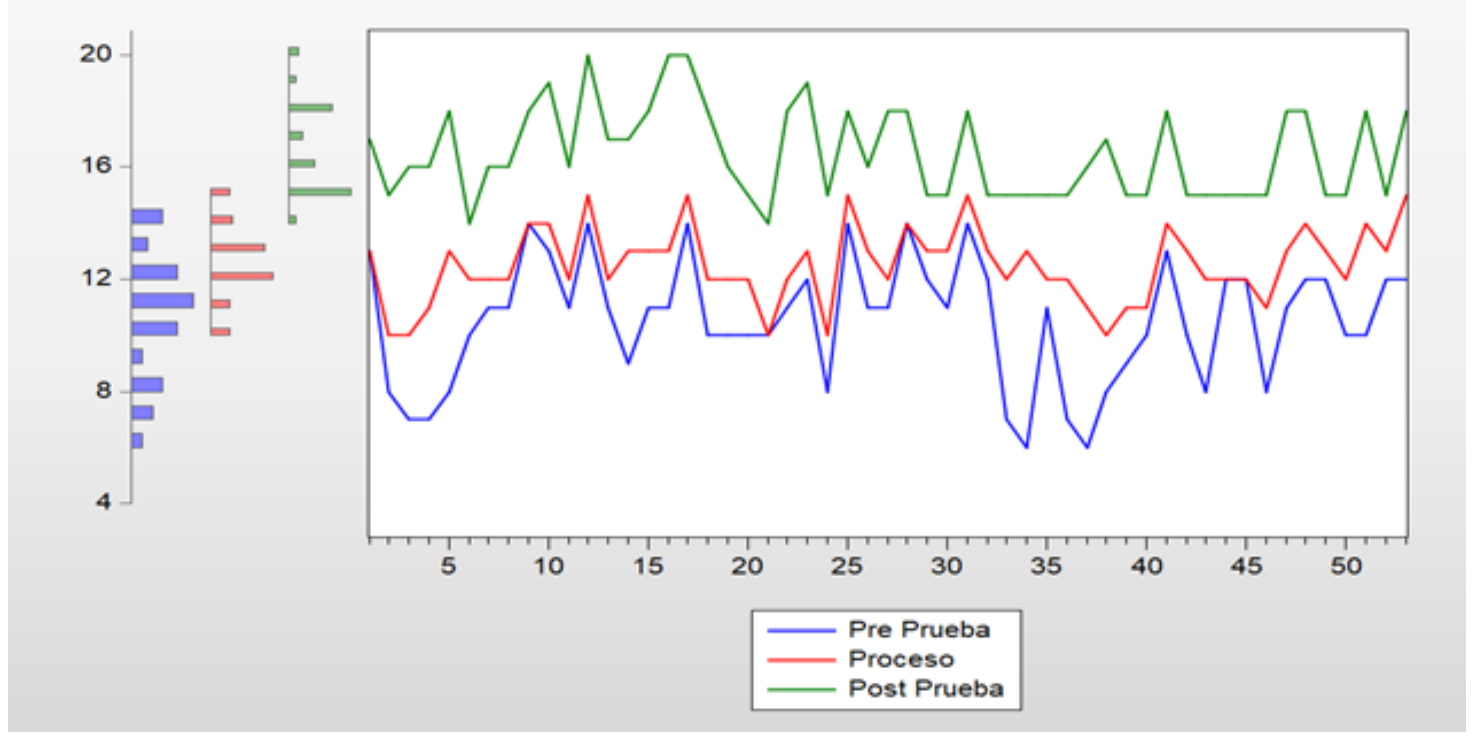

Gráfico 1. Representación gráfica niños según puntuaciones en pre y post prueba.

Previo tratamiento experimental se encuentran ubicados en la escala de proporción que equivale a logro con el $64 \%$, esto implica que los estudiantes lograron desarrollar competencias de grafomotricidad a partir de actividades lúdicas, y desarrollo de un conjunto de actividades tácticas organizadas en talleres de aprendizaje. Finalmente, los efectos de la eficiencia se pueden determinar con una diferencia de 5.9 puntos de diferencia de ambas pruebas. (Tabla 4).

Tabla 4. Resultados de la prueba t test de comparación de medias.

\begin{tabular}{ll}
\hline Variable & Obs Mean Std. Err. Std. Dev. [95\% conf. Interval] \\
\hline P. Salida & 5316.48057 .22638491 .64810716 .0362916 .94484 \\
P. Entrada & 5310.54717 .30065972 .1888369 .94385211 .15049 \\
diff & 535.943396 .2726981 .9847025 .3963456 .490448 \\
\hline
\end{tabular}

Mean $($ diff $)=$ mean $($ P.Salida - P.Entrada $) \mathrm{t}=21.8010$

Ho: mean $($ diff $)=0$ degrees of fredom $=52$

Ha: mean (diff) $<0$ Ha: mean (diff) $!=0$ Ha: mean $($ diff $)>0$

$\operatorname{Pr}(\mathrm{T}<\mathrm{t})=1.0000 \operatorname{Pr}(|\mathrm{T}|>|\mathrm{t}|)=0.0000 \operatorname{Pr}(\mathrm{T}<\mathrm{t})=0.0000$ 
Por tanto, se acepta la hipótesis alterna que la el método y el conjunto de estrategias de la "maestra delivery" ha desarrollado, eficientemente las competencias grafomotoras en estos tiempos de pandemia.

Lo que quiere decir que, en este proceso, existen otras variables exógenas, como el acompañamiento del padre y la madre de familia, la cercanía de los hijos, han ayudado a consolidar mejor el logro de competencias y habilidades de grafomotricidad en sus diferentes dimensiones.

La prueba Dunnet; entre la pre prueba y post prueba, mide el grado de eficiencia del programa que viene a ser $74.6 \%$, determinados por esta prueba estadística que confirma que ha tenido efectos positivos, con confiabilidad estadística del 95\%, aceptables en las investigaciones de las ciencias sociales. (Tabla 5 ).

Tabla 5. Resultados de proporción de Dunnet de comparación entre la pre y post prueba.

P. Entrada
Unadjusted

Contrast Std. Err [ 95\% Conf. Interval ]

\section{Discusión}

La Educación Inicial en el Perú se concibe como el nivel que sienta las bases para la formación integral de los niños menores de 6 años, articulada de manera pedagógica y curricular con la educación primaria (MINEDU, 2017); dedica buena parte del tiempo lectivo, a dar sentido y significado al desarrollo de las competencias comunicativas, en todas sus manifestaciones. Uno de los objetivos principales de la educación inicial constituye la alfabetización (Lara y Pulido, 2020), la adquisición y dominio de la lengua escrita (Gutiérrez y Díez, 2015), que se ha convertido en prerrequisito para la promoción escolar.

La propuesta del MINEDU, en tiempos de pandemia, realmente fracasó, peor su programa estratégico "Aprendo en Casa", diseñado desde el escritorio y desde Lima, no viendo la realidad del Perú profundo. Una vez más queda demostrado en las propuestas y respuestas a la pandemia o política de confinamiento por el COVID-19. Se ha apelado, desde las redes educativas de maestras de inicial, al ingenio y la formación pedagógica de la docente en carrera. En fin, cada maestra trata de recrear una infinidad de estrategias para encarar el problema de la conectividad y al acceso de las herramientas tecnológicas. Se presenta esta propuesta pedagógica, a través de un proceso experimental donde se aplicaron otras experiencias exitosas. Se configuró un escenario donde se sustenta el fundamento epistemológico, incluso esta vivencia pedagógica se puede compartir como estrategia en otros espacios, para validarla. La estrategia de la "maestra delivery" con la promoción de competencias y habilidades grafomotrices en niños en edad preescolar se sustenta en soportes teóricos pedagógicos que son antecedentes de la presente investigación.

Según García Hernández y Batista García (2018) la motricidad fina comprende actividades del niño y la niña que necesitan de una precisión y un elevado nivel de coordinación, los pequeños movimientos que se producen en las manos, muñecas, dedos se da 
generalmente en coordinación con los ojos e implica un nivel elevado de maduración y aprendizaje.

La motricidad fina se refiere a los movimientos de la pinza digital y pequeños movimientos de la mano y muñeca. La adquisición de la pinza digital así como de una mejor coordinación ósculo manual (la coordinación de la mano y el ojo) constituyen uno de los objetivos principales para la adquisición de habilidades de la motricidad fina (Cándales, 2012). Consecuentemente, juega un papel central en el aumento de la inteligencia, promueve el aprendizaje de la escritura. Esta actividad simbólica expresiva caracteriza la cultura y de ser estimulada adecuadamente permite trazar un itinerario de esta capacidad humana por lo que es necesario incentivarla desde las edades más tempranas del Preescolar.

Afrontar con éxito el aprendizaje y el inicio de la alfabetización con niños preescolares, implica para los docentes renovar los procesos pedagógicos habituales, establecer relaciones más cercanas, dialógicas y afectivas con los estudiantes, padres de familia y la comunidad en general, trabajando articuladamente para concretar las aspiraciones relacionadas con la calidad de los aprendizajes y la educación, a través de estrategias innovadoras poco viables a través del internet, como el aprendizajeservicio, que según Puig et al. (2011), se trata de una "propuesta educativa que vincula de una manera circular la participación en servicios pensados para satisfacer alguna necesidad de la comunidad y el aprendizaje de conocimientos y valores" (p. 1).

Frente a la coyuntura actual que se vive en pandemia, se planteó la educación virtual, a partir de Viñals y Cuenca (2016), quienes sostienen que la era digital que ha transformado la educación en un proceso basado en el caos, el cambio y la inestabilidad.
En tal sentido, se pudo observar que los estudiantes en la región Puno y en el distrito de Ilave, no pudieron acceder a la educación virtual planteada por el Ministerio de Educación, debido a la falta de conectividad, acceso a los medios de comunicación y carencia de recursos económicos que presentaban las familias.

De este modo, ante esta situación, se abren paso otras estrategias o formas más democráticas y respetuosas de enseñanza, conintervención conjunta de educadores, comunidad educativa y la familia de los niños para promover capacidades comunicativas funcionales en todas sus manifestaciones, como base de un desarrollo integral (Camacho, 2012).

Se discute así en el presente estudio una propuesta de aprendizaje sustentada en acciones auténticas-participativas y experiencias del ámbito escolar y familiar, para atender articuladamente, las necesidades de aprendizaje de los niños y satisfacer las expectativas de la comunidad, y juntos contribuir a la transformación de nuestros contextos escolares, culturales y sociales.

En este sentido, se planteó el trabajo de investigación denominado la "maestra delivery" como estrategia de aprendizaje-servicio que involucra la participación familiar que posibilita el desarrollo de la motricidad fina de los niños en edad preescolar de las Institución Educativa Inicial.

El modelo de "maestra delivery" como estrategia de aprendizaje-servicio es eficiente $y$ eficaz. A partir del desarrollo de los talleres de aprendizaje, se logró el desarrollo de las habilidades y competencias grafomotrices finas en tiempos de confinamiento. Determinado con el $74 \%$ por medio de la prueba Dunnet, en este proceso coadyuvó de manera extraordinaria el papel de los padres de familia. Así mismo se logra que el niño realice por cuenta propia, acciones válidas y correctas de 
ejecución gráfica en el espacio que debe representar, manteniendo control postural adecuado, dominio del brazo y la manera cómo debe sostener el lápiz, y los movimientos finos que realiza con el brazo en las hojas en blanco que se le facilita, monitoreado por sus padres y asistida por la "maestra delivery" que visita de casa en casa. El procedimiento implicado es próximo a los resultados de otros estudios, se vincula estrechamente las dimensiones de servicio y aprendizaje (Opazo, 2015) y contribuye a materializar ideas que sugieren actividad, creatividad, colaboración, toma de decisiones y responsabilidad en los niños para configurar su estilo de vida (Camacho, 2012).

El niño al desarrollar el sentido de los movimientos y las representaciones de manera autónoma y reflexiva, no se expone la clásica frase de: qué se puede y qué no se puede. Deja la imitación como procedimiento favorito en la educación preescolar, y se predispone por iniciativa propia y estímulo social desarrollar competencias propias y diversificadas que lo identificarán como persona única y distinta. Además, entre las maestras la estrategia de aprendizaje-servicio favorece el desarrollo profesional al enriquecer y compartir dilemas didácticos y propósitos formativos $y$ desarrollo de competencias grafomotrices (Jiménez, 2009) y se constituye en condición necesaria para promover la participación de los miembros de la comunidad, lo cual supone un reto debido a las prácticas pedagógicas que imperan en las escuelas (Sánchez, 2017).

\section{CONCLUSIONES}

La escasa conexión de la institución educativa con la realidad circundante es la mayor limitante para incorporar y articular el aprendizaje-servicio en los aprendizajes curriculares, situación que implica reorientar la organización escolar, establecer proyectos educativos considerando la participación de los coprotagonistas, de manera que las actividades educativas se enriquezcan en el contexto real, haciéndose sostenibles en el tiempo y coadyuven a la concreción de los propósitos educativos. Se considera que el Ministerio de Educación debería incorporar esta estrategia eficiente para el desarrollo de la grafomotricidad en niños en edad pre escolar.

\section{REFERENCIAS}

Arnaíz, P., y Bolarín, M.J. (2016). Introducción a la psicomotricidad. Editorial Síntesis S. A

Camacho, C. (2012). La grafomotricidad en el nivel inicial. Revistas.Unitru.Edu.Pe, 11

Cándales, R. (2012). La capacidad pedagógica para desarrollar la motricidad fina en los niños de 3 a 6 años del Centro de Educación Nacional Bolivariano "El Llano". EduSol, 12(39), 61-71

Chiva-Bartoll, Ó., y Gil-Gómez, J. (2018). Aprendizaje-servicio universitario: modelos de intervención e investigación en la formación inicial docente. Ediciones Octaedro

García Hernández, M., y Batista García, L. M. (2018). El desarrollo de la motricidad fina en los niños y las niñas de la primera infancia. Atlante Cuadernos de Educación y Desarrollo, (agosto)

Gerde, H.K., Wright, T.S., y Bingham, G.E. (2019). Creencias de los maestros de preescolar e instrucción para la escritura. Revista Educación Docente de la Primera Infancia, 40(4), 326-351

Gil, J., Moliner, O., Chiva, O., y López, R. (2016). Una experiencia de aprendizaje-servicio en futuros docentes: Desarrollo de la competencia social y ciudadana. Revista Complutense de Educación, 27(1), 53-73

Gonzáles, J. (2015). Las competencias de comunicación en el inicio de la lectoescritura. Revista Brasileira de Estudios Pedagógicos, 96(243), 416-438 https://bit.ly/3mLZgxP

Gutiérrez, R., y Díez, A. (2015). Aprendizaje de la escritura y habilidades de conciencia fonológica en las primeras edades. Bordón. Revista de Pedagogía, 67(4), 43-59.https://bit.ly/3gcTQJI 
Huda, I., Yulisman, H., Evtia Nurina, C., Erni, F., y Abdullah, D. (2018). Investigating pre-service teachers about their competencies, experiences, and attitudes towards technology integration. Journal of Physics: Conference Series, 114(1, 7). Doi :10.1088/1742-6596/1114/1/012033

Jiménez, C. (2009). "Desarrollo De La Competencia Grafomotora Para La Escritura." Revista Digital Innovación $t$ Experiencias Educativas., 1-12

Lara, P.A., y Pulido, O. (2020). Escritura como práctica de sí y escuela rural. Praxis \& Saber, 11(25), 21-45. https://bit.ly/33NqRat

Mayor, D., y Rodríguez, D. (2015). AprendizajeServicio: Construyendo Espacios De Intersección Entre La Escuela-ComunidadUniversidad. Profesorado. Revista de Currículum y Formación Del Profesorado, 19(1989-639x), 262-279

Mendía, R. (2012). El aprendizaje-servicio : una metodología para la innovación educativa. Aprendizaje Sercicio y Convivencia CONVIVES $16,20-26$

MINEDU. (2017). Programa Curricular de Educación Básica. Programa Curricular de Educación Secundaria, 259. Retrieved from http://repositorio.minedu.gob.pe/ handle/123456789/4550
Opazo, H. (2015). Experiencias de aprendizajeservicio en la formación del profesorado . Un estudio de caso

Puig Rovira, J. M., Gijón Casares, M., Martín García, X., y Rubio Serrano, L. (2011). Aprendizajeservicio y Educación para la Ciudadanía

Puig, J. M., Batlle, R., Bosch, C., y Palos, J. (2007). Aprendizaje servicio. Educar para la ciudadanía. Barcelona: Octaedro

Puig, J. M., y Palos, J. (2006). Rasgos pedagógicos del aprendizaje-servicio. Cuadernos de Pedagogía, 60-63

Sánchez, R. (2017). Desarrollo de la grafomotricidad en niños de 5 años en dos Instituciones Educativas del Distrito de Los Olivos - 2017. Retrieved from http://repositorio.ucv.edu.pe/bitstream/ handle/20.500.12692/22340/montenegro_ rs.pdf? sequence $=1 \&$ is Allowed $=y$

Suárez, B. (2004). El desafío de la escritura: en busca de la grafomotricidad. Revista Iberoamericana de Psicomotricidad y Técnicas Corporales, (16), 5-16

Viñals Blanco, A., y Cuenca Amigo, J. (2016). Revista Interuniversitaria de Formación. Revista Interuniversitaria de Formación Del Profesorado, 30, 163-166. Retrieved from http://www. redalyc.org/pdf/274/27447325012.pdf 